\title{
A compact gene cluster in Drosophila: the unrelated Cs gene is compressed between duplicated amd and $D d c$
}

\author{
Andrey Tatarenkov*, Alberto G. Sáez, Francisco J. Ayala \\ Department of Ecology and Evolutionary Biology, University of California, Irvine, CA 92697-2525, USA
}

Received 16 June 1998; received in revised form 5 February 1999; accepted 10 February 1999; Received by A. Bernardi

\begin{abstract}
$C s$, a gene with unknown function, and amd and $D d c$, which encode decarboxylases, are among the most closely spaced genes in D. melanogaster. Untranslated $3^{\prime}$ ends of the convergently transcribed genes $C s$ and $D d c$ are known to overlap by 88 bp. A number of questions arise about the organization of this tightly-packed gene region and about the evolution and function of the Cs gene. We have now investigated this three-gene cluster in Scaptodrosophila lebanonensis (which diverged from D. melanogaster 60-65 MYA), as well as in D. melanogaster and D. simulans. Gene order and direction of transcription is the same in all three species. The Cs gene codes, in Scaptodrosophila, for a polypeptide of 544 amino acids; in D. melanogaster, it consists of 504 amino acids, which is twice as long as previously suggested, which makes the gene density even more spectacular. The $C s$ sequences exhibit higher number of non-synonymous substitutions between species, higher ratios of non-synonymous to synonymous substitutions, and lower codon usage bias than other genes, suggesting that $C S$ is less functionally constrained than the other genes. This is consistent with the failure of inducing phenotypic mutations in D. melanogaster. The function of Cs remains to be identified, but a high degree of similarity indicates that it is homologous to genes coding for a corticosteroid-binding protein in yeast and a polyamine oxidase in maize. (C) 1999 Elsevier Science B.V. All rights reserved.
\end{abstract}

Keywords: Decarboxylases; D. melanogaster; Gene cluster; Gene duplication

\section{Introduction}

The $D d c$ gene cluster in D. melanogaster, located on the left arm of the second chromosome, includes 18 identified genes plus three transcription units for which no detectable phenotypic mutations are known (Maroni, 1993; Wright, 1996; Stathakis et al., 1995). Most of the genes are densely clustered in two subclusters. Many genes in the cluster are functionally related in that they are involved in the catecholamine metabolism.

Two genes from the proximal subcluster, $D d c$ and amd, have been well studied, with about 90 phenotypic isolated mutations (Wright, 1996). Four genes from the proximal subcluster, including $D d c$ and $a m d$, have been

Abbreviations: amd, $\alpha$-methyl dopa sensitive gene encoding decarboxylase related enzyme (product unknown); bp, base pair(s); BLAST, basic local alignment search tool; $C s$, a gene with unknown function; $D d c$, gene encoding Dopa decarboxylase (DDC, EC 4.1.1.26); ENC, effective number of codons; Myr, million years; MYA, million years ago; PCR, polymerase chain reaction; PLP, pyridoxal 5'-phosphate.

* Corresponding author. Fax: + 1-949-824-2474.

E-mail address: antatare@uci.edu (Andrey Tatarenkov) sequenced in D. melanogaster (Eveleth et al., 1986; Marsh et al., 1986). The coding regions of these two genes are highly similar and are thought to have arisen by gene duplication (Eveleth and Marsh, 1986). An enigmatic gene, called $C s$, lies between amd and $D d c$ (Eveleth and Marsh, 1987). All three genes are among the most closely spaced genes in D. melanogaster, and the $3^{\prime}$ ends of the $D d c$ and $C s$ genes actually overlap by 88 bp (Spencer et al., 1986a; Stathakis et al., 1995). In contrast to $D d c$ and $a m d$, no phenotypic mutations are known for $C s$. The product of the $C s$ gene is not known, although its transcripts have been found associated with polysomes (Spencer et al., 1986b).

$D d c$ has been sequenced in a number of organisms, from mammals to insects, including $D$. melanogaster. Until now, the amd has been studied only in D. melanogaster, and the $C s$ gene is only known to occur in $D$. melanogaster. While $D d c$ and amd are members of a large family of genes, coding for PLP decarboxylases (Jackson, 1990), no genes have been reported that are similar to $C s$. The origin of $C s$ is unknown. Its position between amd and Ddc could be a consequence of the 
original amd-Ddc duplication; or it may have been inserted there at a later time (Eveleth and Marsh, 1987).

Thus, a number of questions arise about $C s$ and its function and location within a developmentally important gene cluster. The first question is whether the $C s$ is present between amd and $D d c$ in other species as well, as it is in D. melanogaster. Second, the compactness of the $C s$, amd, and Ddc cluster in D. melanogaster is unusual, and it is of interest to find out whether this is a result of recent events, or, rather, whether such compactness is old, perhaps tracing back to the time of the amd $-D d c$ duplication. One more question concerns the functional role of the Cs. As $\mathrm{Li}$ (1997, p. 185) has pointed out, it is well known "that the stronger the functional constraints on a macromolecule, the slower the rate of evolution". Thus, if the $C s$ has a less vital function for the organism than amd and $D d c$, it is expected that its evolution be faster than that of the two neighboring genes. Moreover, investigating the pattern of substitutions could help to ascertain whether the $C s$ is a protein encoding gene, which has been questioned (Eveleth and Marsh, 1987).

We have sequenced the $C s$ gene, as well as the whole amd-Cs-Ddc cluster, in the Drosophilid Scaptodrosophila lebanonensis, from a genus closely related to Drosophila. We have also sequenced in $D$. melanogaster Ddc and the coding region of $C s$ in order to resolve inconsistencies arising from previous published sequences. Finally, we have also sequenced most of the three-gene region in $D$. simulans for the purpose of confirming inferences about $D$. melanogaster.

Comparison between the $C s$ genes of $S$. lebanonensis and $D$. melanogaster shows high sequence similarity between them, comparable with the similarity observed for the neighboring $D d c$ and amd genes. Moreover, the regions of high similarity in the nucleotide and putative amino acid sequences extend much beyond the coding region previously suggested for $C s$ (Eveleth and Marsh, 1987). It follows that the three genes are even more tightly packed than had been previously thought for $D$. melanogaster, and that they are partially overlapping.

\section{Materials and methods}

\subsection{Species}

Isofemale lines of Drosophila melanogaster, $D$. simulans, and the closely related Drosophilid Scaptodrosophila lebanonensis were studied. D. melanogaster and D. simulans were collected by one of us (FJA) in St. Lucia, West Indies, in 1995. The strain of $S$. lebanonensis is from the National Drosophila Species Stock Center in Bowling Green, Ohio.

\subsection{DNA preparation and sequencing}

Total genomic DNA was obtained using the phenolchloroform extraction procedure described by Palumbi et al. (1991). To design amplification primers, we compared published sequences of $D d c$ from the moth Manduca sexta (GenBank accession number U03909), the mosquito Aedes aegypti (U27581), and D. melanogaster (X04661), as well as the amd from D. melanogaster (X04695). Ddc and amd in D. melanogaster are quite similar to each other in sequence but have different orientation. We selected segments of the aligned sequences, that had high similarity but also specific substitutions in the amd sequence when compared with $D d c$ sequences. The two primers (forward 5'-GAYATYGARCGNGTSATCATGCCKGG-3', and reverse 5'-GAYATYAGYCGNGTSATCAAGCCKGG-3') encompass large parts of $D d c$ and amd as well as the interval between them (Fig. 1). A region of about $5.8 \mathrm{~kb}$ was obtained in several species of Drosophilidae.

PCR reactions were performed in a $100 \mu$ volume of the ExTAKARA buffer containing $2.5 \mathrm{U}$ of ExTAKARA Taq polymerase, $0.5 \mu \mathrm{M}$ each of the forward and reverse primers, $0.2 \mathrm{mM} \mathrm{dNTP}$, and $3 \mu \mathrm{l}$ of genomic DNA. The cycling parameters for the amplification were an initial denaturation at $95^{\circ} \mathrm{C}$ for $5 \mathrm{~min}$ and 31 cycles of the following: denaturation for $30 \mathrm{~s}$ at $95^{\circ} \mathrm{C}$, annealing for $1 \mathrm{~min}$ at $60^{\circ} \mathrm{C}$, and extension for $5 \mathrm{~min}$ at $72^{\circ} \mathrm{C}$ for the first cycle and an extra $3 \mathrm{~s}$ for every subsequent cycle; after 31 cycles the reaction was additionally kept at $72^{\circ} \mathrm{C}$ for $7 \mathrm{~min}$ to complete extension.

The PCR product of $S$. lebanonensis was purified with Wizard PCR preps DNA purification system (Promega Corporation), and cloned using the TA cloning kit (Invitrogen, San Diego, CA). DNA sequencing was partly done by the dideoxy chain-termination technique with Sequenase Version 2.0 T7 DNA polymerase (Amersham Life Sciences Inc., USA) using ${ }^{35}$ S-labeled dATP, and partly with an ABI model 373 autosequencer using Dye Terminator Ready Reaction Kit in accordance with the manufacture protocol (Perkin Elmer) (see Fig. 1). We employed a successive approach for sequencing the region, so that new sequencing primers were designed based on the sequence obtained with previous primers. Both strands were completely sequenced with 34 primers.

Sequences of the $C s$ gene in both $D$. melanogaster and $D$. simulans were obtained by direct sequencing of purified PCR products with an ABI model 377 autosequencer using the Dye Terminator Ready Reaction Kit in accordance with the manufacturer's protocol (Perkin Elmer). Partial sequences of $D d c$ in D. melanogaster and $D$. simulans were obtained from separately constructed clones of these species. The sequences of these clones overlap considerably with the PCR frag- 


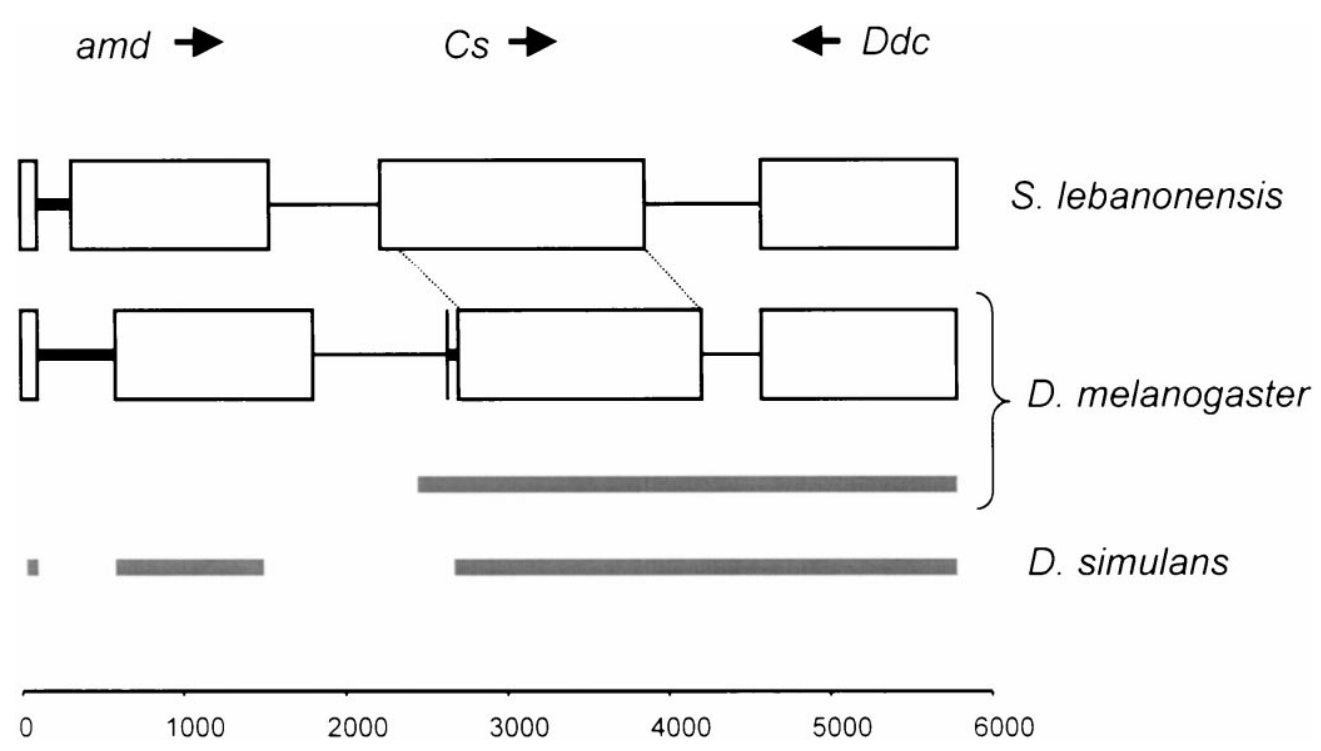

Fig. 1. Structure, gene arrangement, and direction of transcription of a genomic DNA segment comprising the genes amd, Cs, and $D d c$ in Scaptodrosophila lebanonensis and Drosophila melanogaster. Thick arrows adjacent to gene symbols indicate direction of transcription from $5^{\prime}$ to 3'. Boxes indicate protein coding regions: thick lines connecting them represent introns; thin lines represent the non-coding regions. Dotted lines connect the $C s$ regions of high similarity between the two species. The two thick lines in the lower part indicate regions that we have sequenced in D. melanogaster and D. simulans; the rest of the melanogaster sequence is from Marsh et al. (1986) and Eveleth and Marsh (1987). The gene structure and arrangement are the same in D. simulans as in D. melanogaster.

ments. Partial sequence of amd in D. simulans was obtained from yet another clone, which is encompassed by the PCR fragment.

The sequences reported here have been deposited in GenBank database, accession numbers AF091327, AF091328, AF091329, AF121109.

\subsection{Alignment and analysis}

The sequences were edited and assembled using programs of the Fragment Assembly module of the GCG package (Wisconsin Package Version 9.1). Various GCG programs were also used for alignment and translation. Inference about coding regions was primarily obtained by comparison of the $S$. lebanonensis and D. melanogaster sequences seeking regions of high similarity. Additionally, the programs GENIE (Reese et al., 1997) and FGENED (Solovyev et al., 1994) were used for predicting putative exons. Analysis of codon preference was performed with the CODONPREFERENCE program of the GCG package which implements the method of Gribskov et al. (1984). A Fourier transform analysis was performed using the Fast Fourier Transform of the computer program Origin (version 4.10, Microcal Software, Inc.). This method unveils periodicity patterns along binary strings. Such strings were created by using a 1 at each substituted position, and a 0 at identical positions. In addition to the aligned coding regions of $a m d, D d c$, and $C s$ of $D$. melanogaster, $D$. simulans, and $S$. lebanonesis, we also used for illustrative purposes hsr-omega exons 1 and 2 of D. melanogas- ter (U18307) and D. pseudoobscura (X16337). Codonuse bias was assessed by estimating ENC, the 'effective number of codons' (Wright, 1990). Higher values of ENC correspond to lower codon-use bias. Heterogeneity of substitutions along amino acid sequences was tested with the unmodified variance test of Goss and Lewontin (1996). The analysis was kindly conducted by R.C. Lewontin. Rates of substitution at synonymous and non-synonymous sites were calculated by the method of Li (1993). We searched GenBank sequences with the BLAST at http://www.ncbi.nlm.nih.gov/.

\section{Results}

A DNA fragment of approximately $5.8 \mathrm{~kb}$ resulted from PCR amplification in several drosophilid species, Scaptodrosophila lebanonensis, D. melanogaster, D. simulans, D. immigrans, D. mimica, D. (Scaptomyza) palmae, and $D$. (Samoaia) leonensis. The gene organization of the amplified region in D. melanogaster and $S$. lebanonensis is outlined in Fig. 1.

We searched the region between the stop codons of $D d c$ and amd in S. lebanonensis, presumably corresponding to the $C s$ gene, seeking segments similar with the sequence of $C s$ in D. melanogaster (X05991). We found an extended region, about $1.5 \mathrm{~kb}$ with high similarity (71\%) to the sequence of $C s$ in D. melanogaster (Figs. 1 and 2). Unexpectedly, the region of similarity extends more than $400 \mathrm{bp}$ beyond the previously suggested $C S$ stop codon in D. melanogaster (Eveleth and Marsh, 


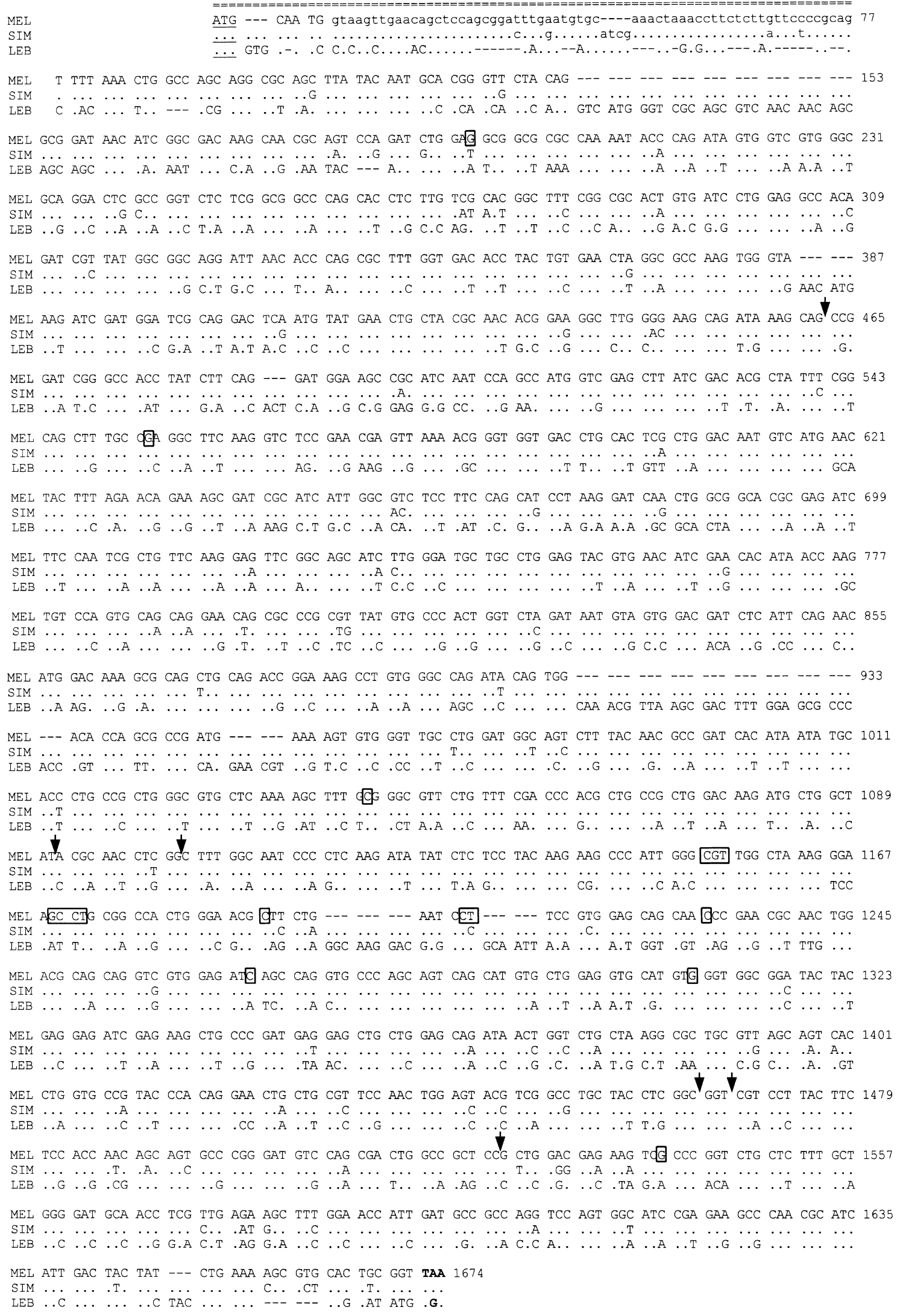

Fig. 2. Alignment of the $C s$ coding region between D. melanogaster (MEL), D. simulans (SIM), and S. lebanonensis (LEB). The intron in D. melanogaster and $D$. simulans is shown in lowercase letters at the top of the figure; the proposed initiator ATGs are underlined; the stop codons are in bold. A region of uncertain alignment is overscored with a double dotted line (top). Dots indicate nucleotides identical to D. melanogaster; hyphens indicate gaps. Discrepancies between our $C s$ sequence and the sequence of D. melanogaster of Eveleth and Marsh (1987) are shown with rectangles to indicate nucleotides missing in their sequence, and arrows to indicate locations at which they show excessive number of nucleotides. 
1987). Moreover, the $S$. lebanonensis sequence has high similarity to a segment upstream of the largest ORF previously identified (Eveleth and Marsh, 1987) in the D. melanogaster $C s$ gene. This whole $1.5 \mathrm{~kb}$ region is an uninterrupted open reading frame (ORF) in $S$. lebanonensis. While the $S$. lebanonensis and D. melanogaster sequences are highly similar at the nucleotide level along the whole $1.5 \mathrm{~kb}$ region, the corresponding peptide sequences are similar only in a few stretches, which are interrupted by stretches that cannot be aligned. This appears to be a consequence of shifts in reading frame due to indels in the published sequence of D. melanogaster (Eveleth and Marsh, 1987) compared with $S$. lebanonensis.

In order to test these inferences, we sequenced the $C s$ gene and adjacent regions in D. melanogaster, as well as in the closely related $D$. simulans. Our $C s$ sequence of $D$. melanogaster differs from the published sequence by the occurrence of nine indels, as predicted by the alignment of the previously published sequence with the $C s$ sequence of $S$. lebanonensis (see Fig. 2).

The corrected sequence of $C s$ in D. melanogaster is very similar to the $C s$ sequence of $D$. simulans. In both species we found a long ORF that extends for $1507 \mathrm{bp}$ from the intron, determined in D. melanogaster by comparison of our genomic sequence with the cDNA sequence of Eveleth and Marsh (1987). The longest ORF previously proposed is $735 \mathrm{bp}$. Thus, the coding region of $C s$ is twice as long as previously thought (Eveleth and Marsh, 1987). In addition, the putative amino acid sequence differs from the one previously suggested for $D$. melanogaster in several stretches, some as long as 30 amino acids. The $C s$ stop codons of the three species are in corresponding positions on our aligned sequences, although several gaps are necessary in order to obtain the alignment (Fig. 2). The alignment of the encoded peptide sequences obtained by translating the ORF yields $95 \%$ amino acid identity between $D$. melanogaster and D. simulans, and $78 \%$ between Scaptodrosophila and those two species.

Although the similarity of the inferred coding regions is high, this high similarity does not start from the very beginning of the coding region. We are thus unable to use sequence comparisons between $D$. melanogaster and $S$. lebanonensis for elucidating the whole length of the coding regions. This is not surprising because the coding segment of the first exon in D. melanogaster is very short, just three codons, according to Eveleth and Marsh (1987). We have used several methods to infer the start of the coding region in S. lebanonensis, and have applied the same methods also to $D$. melanogaster. The programs GENIE and FGENED both predict an intron on the D. melanogaster sequence as detected by Eveleth and Marsh (1987) by comparing cDNA with genomic DNA. They also predict the first short exon postulated by Eveleth and Marsh (1987). The first eight nucleotides of the first exon merge with the remaining $1507 \mathrm{bp}$ of the long ORF that we have found in D. melanogaster. FGENED suggests that the coding region of $C s$ in $S$. lebanonensis consists of just a single exon, which starts 21 codons upstream of the region where similarity between $D$. melanogaster and $S$. lebanonensis can be detected. GENIE yields the same start and stop codons as FGENED. However, GENIE indicates the presence in Scaptodrosophila of a short intron (positions 587718 in Fig. 2). We rather assume that this is a coding segment given that it is highly similar to the sequences of $D$. melanogaster and $D$. simulans along the segment's whole length at both the nucleotide and the amino acid level. It is also possible that the $C s$ in $D$. melanogaster consisted of a continuous single exon in the past, and that an intron ( $62 \mathrm{bp}$ ) may have arisen due to mutations that have disrupted the beginning of the coding sequence. This would explain the somewhat unusual position of the intron, after an exon of only three codons. It is also possible, but seems less likely, that an intron in the ancestral species may have become a coding sequence in $S$. lebanonensis as a result of mutation in the intron's splice site. The predicted peptide length of $C s$ in D. melanogaster is 504 amino acids, compared with 544 amino acids in $S$. lebanonensis, if we assume a single exon.

The regions suggested as protein coding regions are characterized by somewhat increased codon bias along their length (not shown), which is indicative of coding regions (Gribskov et al., 1984). Fig. 3 shows the effective number of codons, ENC, for six genes, including $C s$ and the flanking amd and $D d c$ genes, in the three species, $S$. lebanonensis, D. melanogaster, and D. simulans. In all three species, codon-use is less biased for $C s$ than for any of the other genes, although it is rather similar to that for amd $(\mathrm{ENC}=61$ when all codons are evenly used, $\mathrm{ENC}=20$ when only one codon per amino acid is used).

\section{Discussion}

amd, $C s$, and $D d c$ are neighboring genes in D. melanogaster (Eveleth and Marsh, 1986). amd and Ddc are quite similar in nucleotide and amino acid sequences, and are paralogous genes arising from an ancient gene duplication (Eveleth and Marsh, 1986; Wang et al., 1996). $D d c$ has been sequenced in a number of organisms (Tatarenkov et al., 1999), but the amd and Cs sequences have been reported only for $D$. melanogaster. Comparison of the $D d c$ sequences available in GenBank with those of amd from a number of species (our unpublished data) suggests that the duplication of these genes occurred well before the split of Lepidoptera and Diptera and may predate the divergence of Protostoma and Deuterostoma, which occurred more than 600 


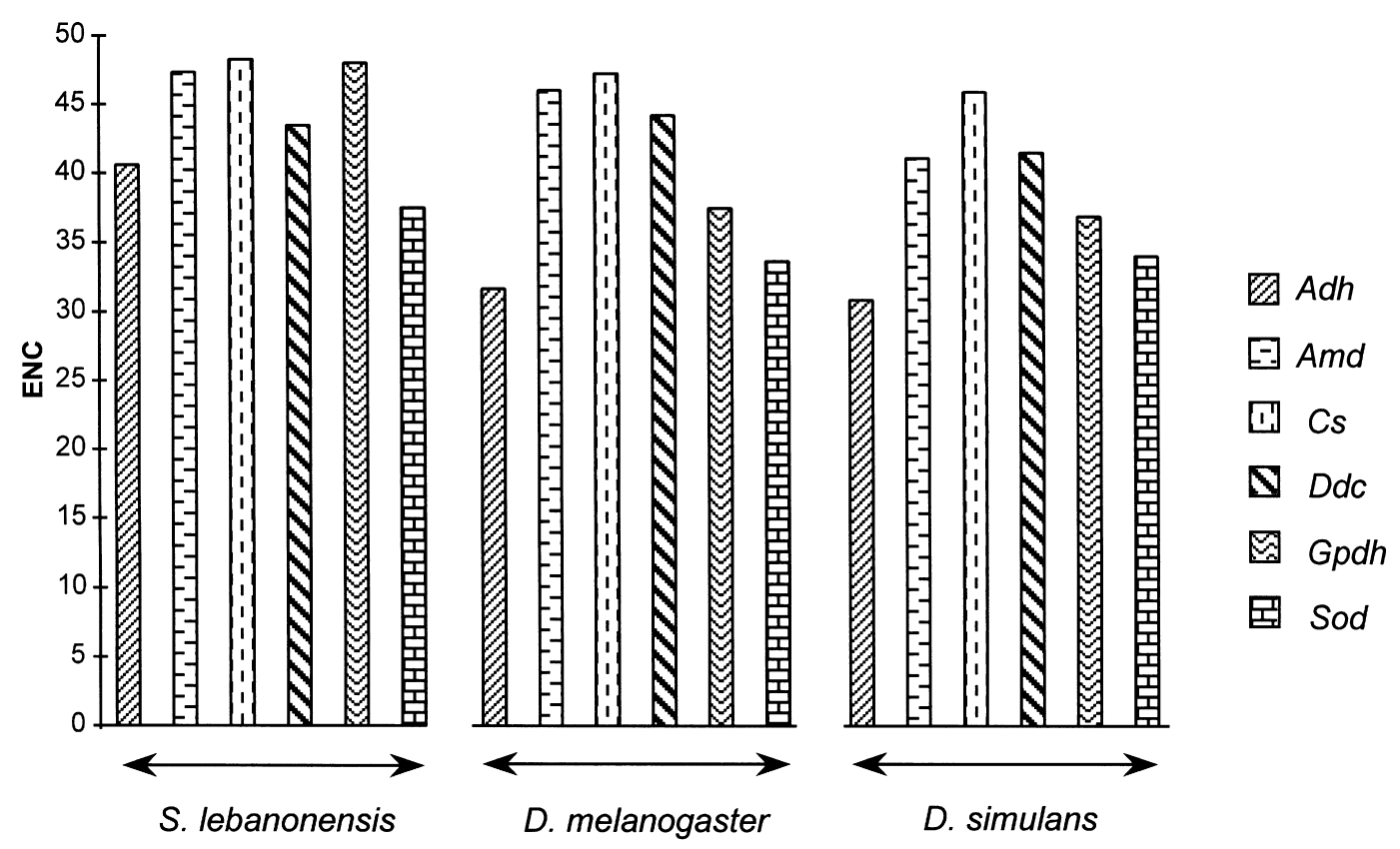

Fig. 3. Codon usage bias in six genes in S. lebanonensis, D. melanogaster, and D. simulans. A larger effective number of codons (ENC) indicates lesser codon usage bias.

MYA, before the Cambrian (Jackson, 1990). If this inference is correct, amd should be present in many animal phyla, unless it has been obliterated, or has evolved beyond recognition. The physical proximity of amd and $D d c$ most likely traces back to the time of the original duplication of these genes, but the presence of Cs between them is enigmatic. It could reflect the survival by a gene that was contiguous to the duplicated gene that led to $D d c$ and $a m d$, or it may have been inserted there at a much later time. The position in diverse animal groups of the orthologous genes to these three might permit us to resolve this issue.

We have amplified and sequenced a $5.8 \mathrm{~kb}$-long fragment of genomic DNA comprising partially the flanking $D d c$ and amd genes and an intermediate region in $D$. melanogaster, D. simulans, and S. lebanonensis, a drosophilid species which diverged from D. melanogaster about 60-65 MYA (Kwiatowski et al., 1994, 1997). A PCR fragment of similar length was obtained from several other Drosophilids. The fact that the region has remained unchanged in several independent lineages during the last 30-40 Myr may be indication of its functional importance. The comparison of the region between amd and Ddc in S. lebanonensis and D. melanogaster has revealed the presence of the $C s$ gene in $S$. lebanonensis, as it was already known in D. melanogaster (Eveleth and Marsh, 1987). Moreover, this Cs gene is also present in $D$. simulans, where the sequence and exon-intron arrangement is extremely similar to our sequence of $D$. melanogaster (but importantly different at a few nucleotide sites from a previously published sequence; see Eveleth and Marsh, 1987). However, it is still not possible to answer when $C s$ arose between amd and $D d c$. Comparisons with species distantly related to Drosophila are necessary, such as remote dipterans, other insects, and Crustacea. These comparisons will also help in dating the time of the ancestral duplication leading to amd and $D d c$. Cs codes for a product of 544 amino acids in $S$. lebanonensis, but 504 amino acids in $D$. melanogaster and $D$. simulans. The larger size than previously proposed of the postulated coding region in D. melanogaster is robust, because in addition to such characteristics of coding regions as increased GC bias and certain codon preferences, the predicted polypeptides exhibit high sequence similarity $(95 \%$ between $D$. melanogaster and D. simulans; $75 \%$ between them and $S$. lebanonensis), which would be unexpected in noncoding regions.

The great proximity of the three genes, amd, $C s$ and $D d c$ in D. melanogaster is quite unusual (see discussion by Eveleth and Marsh, 1987; but see Okuyama et al., 1997). The correct coding region of $C s$ that we have now determined in $D$. melanogaster makes the gene density even more spectacular, with the stop codons of $D d c$ and $C s$ genes being only 366 bp apart. Our study shows that the tight packing also occurs in $S$. lebanonensis, in which the amd stop codon is just $686 \mathrm{bp}$ from the $C s$ start codon, and the stop codons of $C s$ and $D d c$ are only $722 \mathrm{bp}$ apart from one another (Fig. 1). The suggestion that mutagenic silence of the $C s$ may have occurred in D. melanogaster as a consequence of evolutionarily recent modifications in the gene's structure (Eveleth and Marsh, 1987) becomes unconvincing, given that $C s$ has remained tightly packed with $D d c$ and amd 
Table 1

Number of non-synonymous (n-syn) and synonymous (syn) substitutions per site \pm SE, and their ratio (n-syn/syn), between Drosophila melanogaster, D. simulans, and Scaptodrosophila lebanonensis at six nuclear genes. The sequences of Adh are from Russo et al. (1995); Gpdh from Kwiatowski et al. (1997); Sod from Kwiatowski et al. (1994)

\begin{tabular}{|c|c|c|c|c|}
\hline & & melanogaster-simulans & melanogaster-lebanonensis & simulans-lebanonensis \\
\hline \multirow[t]{3}{*}{ Amd } & n-syn & $0.010 \pm 0.004$ & $0.105 \pm 0.013$ & $0.099 \pm 0.012$ \\
\hline & syn & $0.151 \pm 0.027$ & $1.313 \pm 0.174$ & $1.372 \pm 0.188$ \\
\hline & ratio & 0.066 & 0.080 & 0.072 \\
\hline \multirow[t]{3}{*}{$C s$} & n-syn & $0.027 \pm 0.005$ & $0.212 \pm 0.016$ & $0.217 \pm 0.016$ \\
\hline & syn & $0.149 \pm 0.022$ & $1.562 \pm 0.204$ & $1.467 \pm 0.186$ \\
\hline & ratio & 0.181 & 0.136 & 0.148 \\
\hline \multirow[t]{3}{*}{$D d c$} & n-syn & $0.003 \pm 0.002$ & $0.066 \pm 0.010$ & $0.064 \pm 0.010$ \\
\hline & syn & $0.064 \pm 0.018$ & $1.239 \pm 0.175$ & $1.155 \pm 0.156$ \\
\hline & ratio & 0.047 & 0.053 & 0.055 \\
\hline \multirow[t]{3}{*}{$A d h$} & n-syn & $0.002 \pm 0.002$ & $0.101 \pm 0.016$ & $0.103 \pm 0.017$ \\
\hline & syn & $0.052 \pm 0.021$ & $0.802 \pm 0.122$ & $0.765 \pm 0.117$ \\
\hline & ratio & 0.038 & 0.126 & 0.135 \\
\hline \multirow[t]{3}{*}{ Gpdh } & n-syn & $0.000 \pm 0.000$ & $0.012 \pm 0.005$ & $0.014 \pm 0.005$ \\
\hline & syn & $0.060 \pm 0.019$ & $1.296 \pm 0.251$ & $1.194 \pm 0.209$ \\
\hline & ratio & 0.000 & 0.009 & 0.012 \\
\hline \multirow[t]{3}{*}{ Sod } & n-syn & $0.000 \pm 0.000$ & $0.113 \pm 0.020$ & $0.108 \pm 0.019$ \\
\hline & syn & $0.114 \pm 0.037$ & $1.508 \pm 0.473$ & $1.938 \pm 1.030$ \\
\hline & ratio & 0.000 & 0.075 & 0.056 \\
\hline
\end{tabular}

for a considerable time, at least $60-65 \mathrm{Myr}$ in $S$. lebanonensis and D. melanogaster. The structure and sequence of this region have remained essentially identical in D. simulans and D. melanogaster, that is for some 2.5 Myr (we have not investigated the region upstream of the $C s$ coding sequence in $D$. simulans, but it also seems quite similar with respect to length, since the PCR fragments are of similar length).

Eveleth and Marsh (1987) failed to recover Cs phenotypic mutants in their extensive mutagenesis screens and suggested that this implies that the $C$ f function is not essential or that $C s$ RNA does not encode a protein, as amd

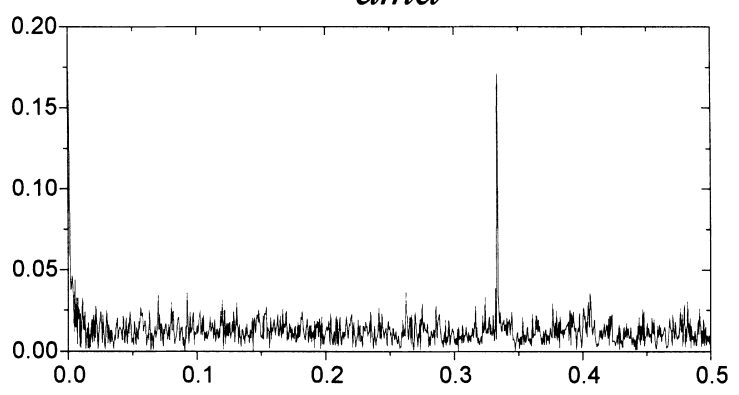

Cs

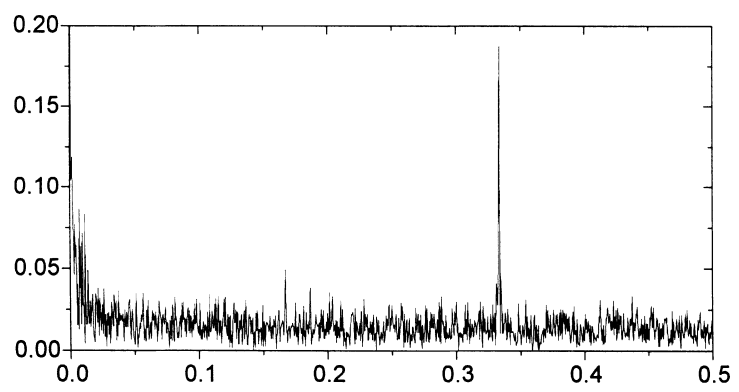

$D d c$

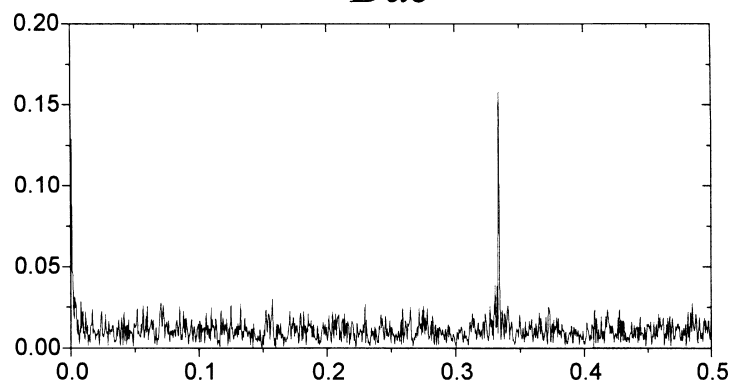

Hsr-omega

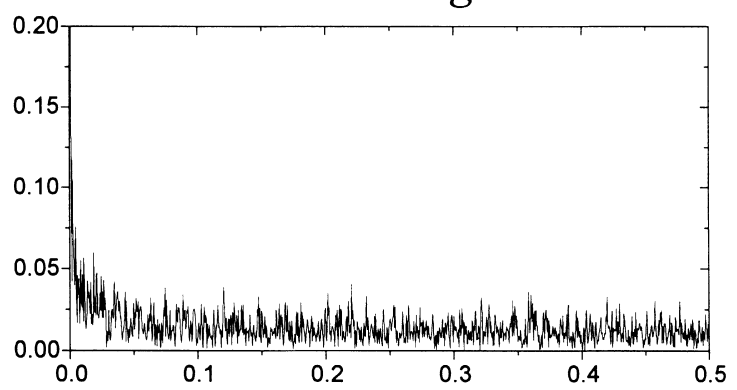

Fig. 4. Fourier transform of the substitution pattern in four genes: amd, Ddc, and Cs are from D. melanogaster, D. simulans, and S. lebanonensis, hsr-omega is from D. melanogaster and D. pseudoobscura. A dominant substitution frequency of $1 / 3$ is revealed for the exon sequences of amd, $D d c$, and $C s$, while no predominant peak is observed for the two exons of the non-coding hsr-omega (a peak very close to the $y$-axis is due to nonspecific correlations and is largely diminished when gaps are eliminated from the alignment; data not shown). 
described for hsr-omega in Drosophila (Fini et al., 1989). We propose, however, that $C s$ retains protein-encoding capacity. Thus, the ratio of non-synonymous to synonymous substitutions between $D$. melanogaster and $D$. simulans, or between the later two and S. lebanonensis, is much less than 1 (Table 1 ). In a complementary analysis (which may be more appropriate for a comparison between distantly related species, such as $S$. lebanonensis with respect to $D$. melanogaster/D. simulans, because of the possible saturation at synonymous sites) the pattern of substitutions also indicates a proteinencoding capacity for $C s$. If so, $C s$ should have a dominant peak of periodical substitutions every third base. Periodicity in DNA sequencies can be unveiled using Fourier analysis (Tsonis et al., 1991), which we have investigated using an algorithm by Cooley and Tukey (1965). A clear dominant substitution frequency of $1 / 3$ (i.e. every third position) is observed for amd, $D d c$, but also for $C s$, while it is not for hsr-omega (Fig. 4), although the percentage of substitutions is similar in the four genes: $27.8 \%, 21.6 \%, 36.6 \%$, and $24.4 \%$, respectively. Nevertheless, there is some indication that the selective constraints may be somewhat lower for $C s$ than for other genes. Thus, the ratio of non-synonymous to synonymous substitutions is higher in $C s$ than in the other five genes (Table 1). Additionally, the number of non-synonymous substitutions per site is higher in Cs. Moreover, several gaps, some as long as 10 codons, are needed to align the $C s$ sequences of the three studied species, whereas only one or three gaps are required in amd and $D d c$.

The hypothesis of lesser functional constraints imposed on $C s$ is furthermore supported by analysis of codon usage bias, which is lowest in $C s$ for all three Drosophilidae that we have studied (Fig. 3). Irrespective of the mechanism underlying the natural selection on silent sites (e.g. rates of protein elongation, translational accuracy), codon usage is typically most biased in highly expressed genes with high functional constraints (Shields et al., 1988; Moriyama and Hartl, 1993; Akashi, 1994; Moriyama and Powell, 1997). Note, however, that although the codon usage bias in $C s$ is not as pronounced as in such highly expressed genes as $A d h$ and $S o d$, it is not untypical for Drosophila. Particularly, ENC in $C s$ is rather close to that in the neighboring amd. Earlier observations that codon usage bias in $C s$ is very weak compared with other D. melanogaster genes (Eveleth and Marsh, 1987; Stathakis et al., 1995) may have arisen from mistakes in the previously published sequence of $C s$.

We have studied the spatial distribution of substitutions in the deduced amino acid sequences of amd, $C s$, and $D d c$ (Fig. 5). The three proteins show seemingly different distribution of the substitutions, with $C s$ appearing as the most homogeneous. However, the unmodified variance test of Goss and Lewontin (1996)
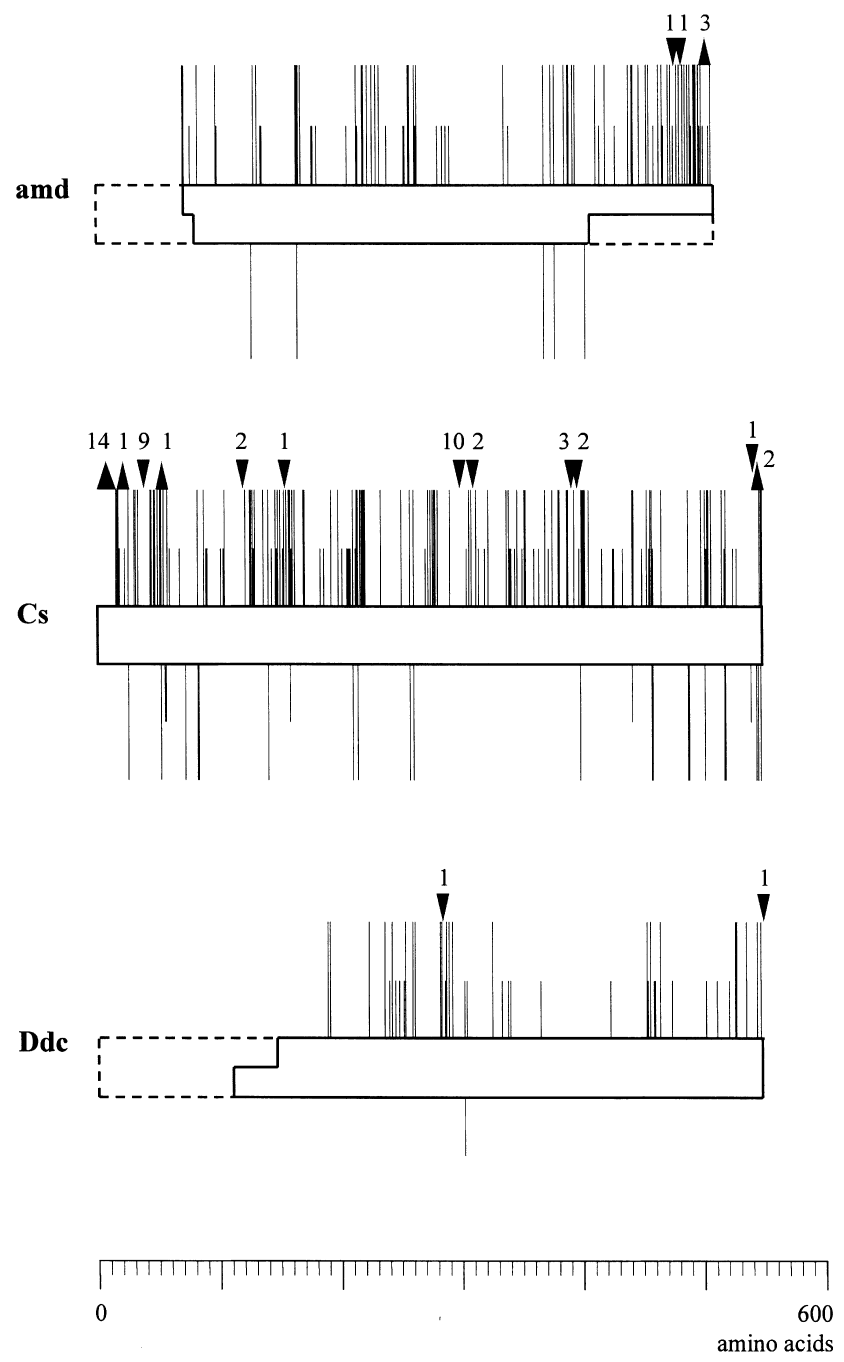

Fig. 5. Amino acid substitutions along amd, $C s$, and $D d c$ between $D$. melanogaster, and D. simulans or S. lebanonensis. The three boxes represent, from top to bottom respectively, the aligned protein sequences of $a m d, C s$, and $D d c$. The upper part of the box corresponds to the alignment between $D$. melanogaster and $S$. lebanonensis, the lower part between D. melanogaster and D. simulans. Dashed lines indicate regions where the alignment was not feasible owing to the absence of at least one sequence. Substitutions are shown by vertical lines: short when the amino acid replacements are conservative $(\mathrm{D} / \mathrm{E}$, $\mathrm{K} / \mathrm{R} / \mathrm{H}, \mathrm{N} / \mathrm{Q}, \mathrm{S} / \mathrm{T}, \mathrm{I} / \mathrm{L} / \mathrm{V}, \mathrm{F} / \mathrm{W} / \mathrm{Y}$, or $\mathrm{A} / \mathrm{G}$, according to Smith and Smith, 1990), and long when they are not conservative (any other substitutions). Arrowheads indicate the position of gaps, pointing down when they occur in D. melanogaster, and up for the compared sequence. Numbers indicate the number of amino acid residues. A scale is at the bottom.

reveals statistically significant non-random clustering of substitutions $(P<0.01)$ in all three genes in the comparison between $D$. melanogaster and $S$. lebanonensis, both including and excluding the conservative substitutions (short lines in Fig. 5). Interestingly, the non-randomness is more pronounced for all three proteins when conservative substitutions are not considered (i.e. $P$ values are smaller). A graphical plot of the distribution of the segment sizes between substitutions shows an excess, 
compared with random distribution, of large segments in $D d c$ and $a m d$, and of contiguous substitutions in $C s$ (data not shown). A remarkable area of low contrained evolution is the carboxyl end of amd, while the central areas of amd and $D d c$ appear to be the most constrained ones. In $C s$ it is difficult to distinguish areas of low interspecific variation, and the non-random distribution of substitutions is probably due to an excess of runs of contiguous substitutions, as mentioned above.

As shown above $C s$ appears to be a protein-encoding gene. Consequently, we have conducted an extensive search of GenBank for sequences that would be similar to $C s$, and have at least six sequences that are distantly related to $C s$, although more similar than expected by chance. Fig. 6 displays the protein alignments with the two most similar sequences: a corticosteroid-binding protein in the yeast Candida albicans, and a polyamine oxidase in maize, Zea mays. Although the similarity of these sequences to $C s$ is not very high, they are surely homologous. First, the probability of the sequence similarity observed is in both cases $P<10^{-6}$. Moreover, the alignment encompasses large segments of the genes: about $90 \%$ of the Cs gene in S. lebanonensis and $95 \%$ in D. melanogaster and virtually the whole extension of the coding regions in the genes of Candida and Zea. Other sequences of about the same length and with similarity nearly as large include amine oxidase in a fish (P49253); protoporphyrinogen oxidase in tobacco (Y13466); and proteins of unknown function with similarities to monoamine oxidase and protein kinase in Caenorhabditis (z78198, locus 1491653) and Arabidopsis (G2244987). No single sequence that is particularly close to the $C s$ gene could be singled out; instead, all these sequences are approximately equally similar to it, suggesting that the split of $C s$ from the common ancestral gene is very ancient, perhaps predating the diversification of the major multicellular kingdoms.

\section{Conclusions}

(1) Gene order and direction of transcription of the amd, $C s$, and $D d c$ genes are the same in S. lebanonensis and $D$. melanogaster. $C s$ is very closely packed with the neighboring $D d c$ and amd genes in S. lebanonensis as well as in Drosophila.

(2) The $C s$ gene codes for a longer product than had been previously suggested for $D$. melanogaster. The length of the deduced protein is 544 amino acids in $S$. lebanonensis and 504 amino acids in D. melanogaster. In $S$. lebanonensis the protein is encoded by a single ORF, while in D. melanogaster the coding sequence is interrupted by a short intron.

(3) There is heterogeneity in substitution pattern between and within amd, $C s$, and $D d c$. $D d c$ appears to be the most constrained gene of the three, especially its central area. amd is less constrained, with a highly variable carboxyl end and a more conserved central area. Cs is affected the most by the substitution process, with runs of contiguous substitutions along its whole length.

(4) Compared with some other nuclear genes, the Drosophilidae $C s$ sequences exhibit higher number of non-synonymous substitutions, higher ratios of nonsynonymous to synonymous substitutions, and lower

\begin{tabular}{|c|c|c|c|c|}
\hline \multicolumn{3}{|c|}{ Corticosteroid-binding protein in yeast (Candida albicans) } & \multicolumn{2}{|c|}{ Polyamine oxidase in maize (Zea mays) } \\
\hline leban: & 55 & 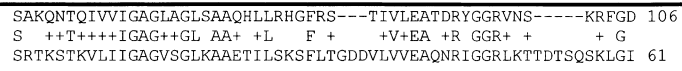 & $\begin{array}{l}\text { leban: } 50 \\
\text { maize: } 23\end{array}$ & 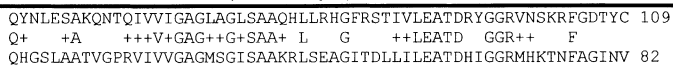 \\
\hline leban: & 107 & 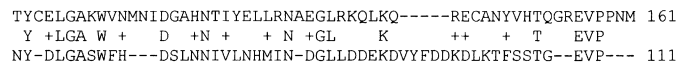 & leban: 110 & 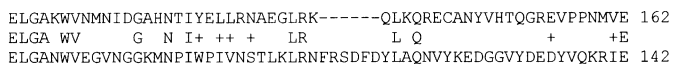 \\
\hline leban: & 112 & 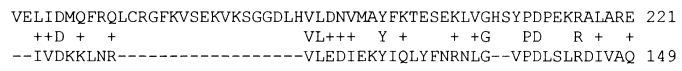 & $\begin{array}{l}\text { leban: } 164 \\
\text { maize: } 143\end{array}$ & 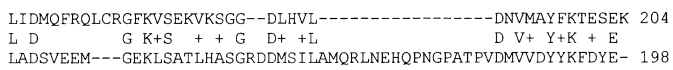 \\
\hline leban: & 222 & 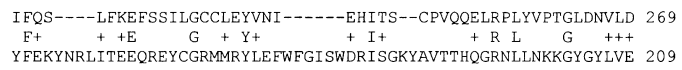 & leban: 205 & 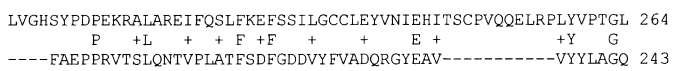 \\
\hline leban: & 270 & 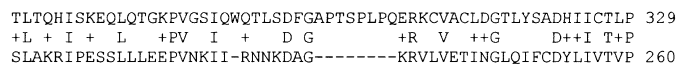 & leban: 265 & 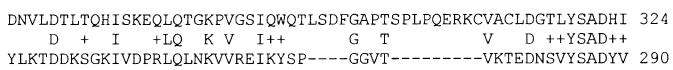 \\
\hline leban: & 330 & 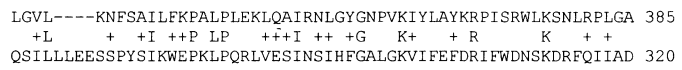 & leban: 325 & 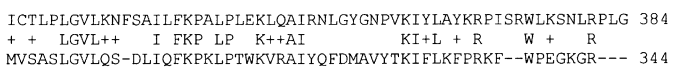 \\
\hline leban: & 386 & 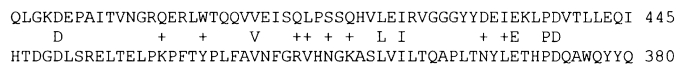 & leban: 385 & 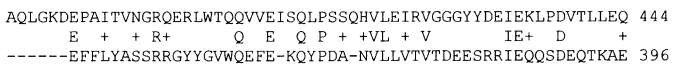 \\
\hline leban: & 446 & 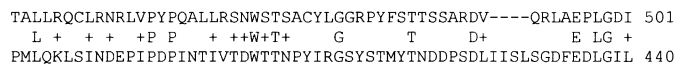 & leban: 445 & 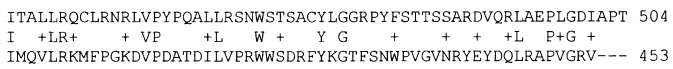 \\
\hline leban: & $\begin{array}{l}502 \\
441\end{array}$ & $\begin{array}{l}\text { APTLLFAGDATALKGFGTIDGARTSGIREAQRIID } 536 \\
\text { P + FAG + T +G G }+ \text { GA SGI A I++ } \\
\text { EPYIKFAGEHTTSEGTGCVHGAYMSGIYAADCILE } 475\end{array}$ & $\begin{array}{l}\text { leban: } 505 \\
\text { maize: } 454\end{array}$ & $\begin{array}{l}\text { LLFAGDATALKGFGTIDGARTSGIREAQRIID } 536 \\
\text { F G+ T+ G+ GA SGI A+ + I+ } \\
\text {-YFTGEHTSEHYNGYVHGAYLGIDSAEILIN } 484\end{array}$ \\
\hline
\end{tabular}

Fig. 6. Similarity between the deduced $C s$ protein in S. lebanonensis and two other proteins: corticosteroid-binding protein in the yeast Candida albicans (PIR: A47259), and polyamine oxidase in maize (GenBank: AJ002204). The numbers at the two ends of each row refer to amino acid sites in the proteins. Identical amino acids are shown by letters in the middle rows; crosses indicate functionally similar amino acids. 
codon usage bias, suggesting that $C s$ is not functionally so highly constrained as the other genes.

(5) The Cs protein exhibits statistically significant sequence similarity to other proteins, such as some oxidases.

\section{Acknowledgements}

We thank R.C. Lewontin for running the unmodified variance test for us; Shiliang Qin and John W. Jacobs for use of an automatic sequencer of the Hitachi Chemical Research Center, Inc. at the University of California, Irvine; and José L. Oliver, Wentian Li, and Hubert Berens for help with the Fourier analysis. Research supported by NIH Grant GM42397 to F.J.A.

\section{References}

Akashi, H., 1994. Synonymous codon usage in Drosophila melanogaster: natural selection and translational accuracy. Genetics 136, 927-935.

Cooley, J.W., Tukey, J.W., 1965. An algorithm for the machine calculation of complex Fourier series. Math. Comput. 19, 297-301.

Eveleth, D.D., Marsh, J.L., 1986. Evidence for evolutionary duplication of genes in the dopa decarboxylase region of Drosophila. Genetics 114, 469-483.

Eveleth, D.D., Marsh, J.L., 1987. Overlapping transcription units in Drosophila: sequence and structure of the Cs gene. Mol. Gen. Genet. 209, 290-298.

Eveleth, D.D., Gietz, R.D., Spencer, C.A., Nargang, F.E., Hodgetts, R.B., Marsh, J.L., 1986. Sequence and structure of the dopa decarboxylase gene of Drosophila: evidence for novel RNA splicing variants. EMBO J. 5, 2663-2672.

Fini, M.E., Bendena, W.G., Pardue, M.L., 1989. Unusual behavior of the cytoplasmic transcript of hsr omega: an abundant, stress-inducible RNA that is translated but yields no detectable protein product. J. Cell Biol. 108, 2045-2057.

Goss, P.J.E., Lewontin, R.C., 1996. Detecting heterogeneity of substitution along DNA and protein sequences. Genetics 143, 589-602.

Gribskov, M., Devereux, J., Burgess, R.R., 1984. The codon usage plot: graphic analysis of protein coding sequences and prediction of gene expression. Nucleic Acids Res. 12, 539-549.

Jackson, F.R., 1990. Prokaryotic and Eukaryotic pyridoxal-dependent decarboxylases are homologous. J. Mol. Evol. 31, 325-329.

Kwiatowski, J., Skarecky, D., Bailey, K., Ayala, F.J., 1994. Phylogeny of Drosophila and related genera inferred from the nucleotide sequence of the Cu,Zn Sod gene. J. Mol. Evol. 38, 443-454.

Kwiatowski, J., Krawczyk, M., Jaworski, M., Skarecky, D., Ayala, F.J., 1997. Erratic evolution of glycerol-3-phosphate dehydrogenase in Drosophila, Scaptomyza, and Ceratitis. J. Mol. Evol. 44, $9-22$.

Li, W.-H., 1993. Unbiased estimation of the rates of synonymous and nonsynonymous substitution. J. Mol. Evol. 36, 96-99.
Li, W.-H., 1997. Molecular Evolution. Sinauer Associates, Sunderland, MA.

Maroni, G., 1993. An Atlas of Drosophila Genes: Sequences and Molecular Features. Oxford University Press, New York.

Marsh, J.L., Erfle, M.P., Leeds, C.A., 1986. Molecular localization, developmental expression and nucleotide sequence of the alphamethyldopa hypersensitive gene of Drosophila. Genetics 114, 453-467.

Moriyama, E.N., Hartl, D.L., 1993. Codon usage and base composition of nuclear genes in Drosophila. Genetics 134, 847-858.

Moriyama, E.N., Powell, J.R., 1997. Codon usage bias and tRNA abundance in Drosophila. J. Mol. Evol. 45, 514-523.

Okuyama, E., Tachida, H., Yamazaki, T., 1997. Molecular analysis of the intergenic region of the duplicated Amy genes of Drosophila melanogaster and Drosophila teissieri. J. Mol. Evol. 45, 35-42.

Palumbi, S., Martin, A., Romano, S., MacMillan, W.O., Stice, L., Grabovsky, G., 1991. The Simple Fool's Guide to PCR, Version 2.0. Department of Zoology and Kewalo Marine Laboratory, University of Hawaii, Honolulu.

Reese, M.G., Eeckman, F.H., Kulp, D., Haussler, D., 1997. Improved splice site detection in Genie. J. Comput. Biol. 4, 311-323.

Russo, C.A.M., Takezaki, N., Nei, M., 1995. Molecular phylogeny and divergence times of drosophilid species. Mol. Biol. Evol. 12, 391-404.

Shields, D.C., Sharp, P.M., Higgins, D.G., Wright, F., 1988. 'Silent' sites in Drosophila genes are not neutral: evidence of selection among synonymous codons. Mol. Biol. Evol. 5, 704-716.

Smith, R.F., Smith, T.F., 1990. Automatic generation of primary sequence patterns from sets of related protein sequences. Proc. Natl. Acad. Sci. USA 87, 118-122.

Solovyev, V.V., Salamov, A.A., Lawrence, C.B., 1994. Predicting internal exons by oligonucleotide composition and discriminant analysis of spliceable open reading frames. Nucleic Acids Res. 22, 5156-5163.

Spencer, C.A., Gietz, R.D., Hodgetts, R.B., 1986a. Overlapping transcription units in the Dopa decarboxylase region of Drosophila. Nature 322, 279-281.

Spencer, C.A., Gietz, R.D., Hodgetts, R.B., 1986b. Analysis of the transcription unit adjacent to the $3^{\prime}$ end of the dopa decarboxylase gene in Drosophila melanogaster. Dev. Biol. 114, 260-264.

Stathakis, D.G., Pentz, E.S., Freeman, M.E., Kullman, J., Hankins, G.R., Pearlson, N.J., Wright, T.R.F., 1995. The genetic and molecular organization of the Dopa decarboxylase gene cluster of Drosophila melanogaster. Genetics 141, 629-655.

Tatarenkov, A., Kwiatowski, J., Skarecky, D., Barrio, E., Ayala, F.J., 1999. On the evolution of Dopa decarboxylase (Ddc) and Drosophila systematics. J. Mol. Evol.. in press

Tsonis, A.A., Elsner, J.B., Tsonis, P.A., 1991. Periodicity in DNA coding sequences: implications in gene evolution. J. Theor. Biol. 151, 232-331.

Wang, D., Marsh, J.L., Ayala, F.J., 1996. Evolutionary changes in the expression pattern of a developmentally essential gene in the three Drosophila species. Proc. Natl. Acad. Sci. USA 93, 7103-7107.

Wisconsin Package Version 9.1, Genetics Computer Group (GCG), Madison, WI.

Wright, F., 1990. The effective number of codons used in a gene. Gene 87, 23-29.

Wright, T.R.F., 1996. Phenotypic analysis of the Dopa decarboxylase gene cluster mutants in Drosophila melanogaster. J. Hered. 87, 175-190. 